HVMUX, the High Voltage Multiplexing for the ATLAS Tracker Upgrade

This content has been downloaded from IOPscience. Please scroll down to see the full text. 2015 JINST 10 C01041

(http://iopscience.iop.org/1748-0221/10/01/C01041)

View the table of contents for this issue, or go to the journal homepage for more

Download details:

IP Address: 131.169.3.59

This content was downloaded on 10/09/2015 at 14:21

Please note that terms and conditions apply. 
Topical Workshop on Electronics for Particle Physics 2014, 22-26 SEPTEMBER 2014, Aix en Provence, France

\title{
HVMUX, the High Voltage Multiplexing for the ATLAS Tracker Upgrade
}

\author{
E. Giulio Villani, ${ }^{a}{ }^{1}$ P. Phillips, ${ }^{a}$ J. Matheson, ${ }^{a}$ Z. Zhang, ${ }^{a}$ D. Lynn, ${ }^{b}$ P. Kuczewski, ${ }^{b}$ \\ L.B.A. Hommels, ${ }^{c}$ I. Gregor, ${ }^{d}$ M. Bessner, ${ }^{d}$ K. Tackmann, ${ }^{d}$ F.M. Newcomer, ${ }^{e}$ \\ E. Spencer ${ }^{f}$ and A. Greenall ${ }^{g}$ \\ ${ }^{a}$ STFC Rutherford Appleton Laboratory Particle Physics Dept., \\ Harwell, Didcot, OX110QX, U.K. \\ ${ }^{b}$ Brookhaven National Laboratory (BNL), \\ Upton, NY, U.S.A. \\ ${ }^{c}$ Cavendish Laboratory, University of Cambridge, \\ Cambridge, U.K. \\ ${ }^{d}$ Deutsches Elektronen Synchrotron (DESY), \\ Hamburg, Germany \\ ${ }^{e}$ University of Pennsylvania, \\ Philadelphia, PA, U.S.A. \\ ${ }^{f}$ University of California Santa Cruz (SCIPP-UCSC), \\ Santa Cruz, CA, U.S.A. \\ ${ }^{g}$ Oliver Lodge Laboratory, The University of Liverpool, \\ Liverpool, U.K. \\ E-mail: giulio.villani@stfc.ac.uk
}

ABSTRACT: The increased luminosity of the HL-LHC will require more channels in the upgraded ATLAS Tracker, as a result of the finer detector segmentation. Thus, an upgraded and more efficient HV biasing of the sensors will also be needed and is among the many technological challenges facing the ATLAS Tracker Upgrade. A number of approaches, including the sharing of the same HV line among several sensors and suitable HV switches, along with their control circuitry are currently being investigated for this purpose. The proposed solutions along with latest test results and measurements will be described.

KEYWORDS: Voltage distributions; Radiation-hard electronics; Large detector systems for particle and astroparticle physics

\footnotetext{
${ }^{1}$ Corresponding author.
} 


\section{Contents}

1 Introduction 1

2 HV MUX system description 1

2.1 Devices irradiation test description 3

2.2 Stacked configuration for Higher Voltages 5

3 Conclusions $\quad 6$

\section{Introduction}

The current Semiconductor Tracker system (SCT), one of the three sub-detectors of the present ATLAS tracker, provides the High Voltage biasing to the strip sensors by means of individual High Voltage supplies, which are remotely located in a service cavern [1,2].

This one module - one PSU approach represents an optimal solution in terms of robustness, as this simplifies the operation of disabling malfunctioning sensors without disturbing the others. It also allows easy monitoring of the individual current drawn by each sensor, an important requisite to assess their proper functioning.

However, owing to the increased number of sensors in the Tracker Upgrade, it will be not feasible to use the existing HV conductors to implement the individual biasing approach: lack of space for cables and traces will not permit each sensor to have its own HV bias [3]. For instance, if existing HV cables are re-used, groups of 4 sensors or more will need to be connected in parallel and this would lead to the loss of the other modules on the same bias line, should one sensor fail to high current. In order to avoid such potential losses, the approach currently being investigated consists of having all the 13 modules on one side of the stave powered by a single HV bias line. Each sensor can in turn be disconnected from the bias line with a DCS controlled HV multiplexer switch (MUX).

In this paper, a detailed description of the proposed HV MUX approach will be provided. Description of a test system that allows real time monitoring of the electrical characteristics of HV devices during irradiation will also be given, along with the latest test results of the investigated devices. Next, a description and test results of a circuitry solution that allows switching of high voltages using lower voltage rated devices will be given and finally preliminary conclusions along with next planned steps of the project will be given.

\section{HV MUX system description}

The ATLAS Tracker Upgrade will consist of an all silicon tracking detector, to fulfill the requirements of the High Luminosity upgrade of the Large Hadron Collider (HL-LHC) [4, 5]. The baseline solution for the strip barrel is the stave concept, in which a number, up to 13 , of single sided silicon 


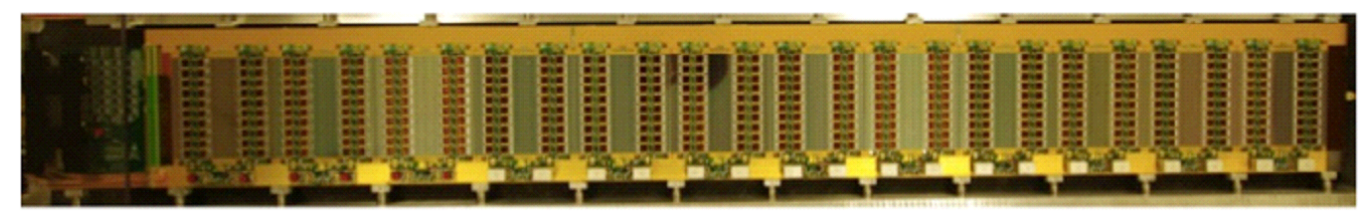

Figure 1. A stave 250.

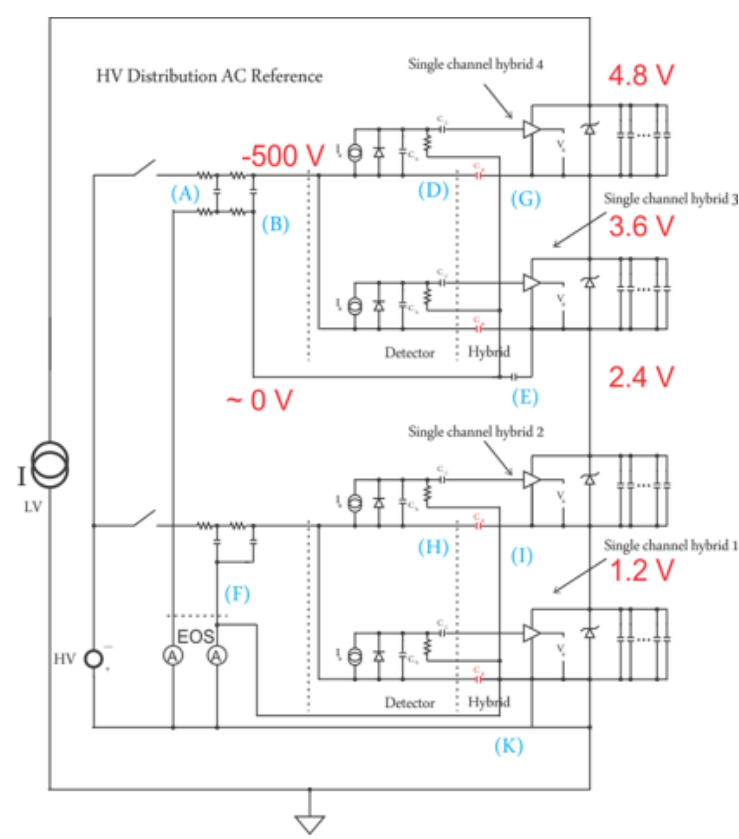

Figure 2. A simplified HV MUX schematic diagram in a SP scheme. The HV switches, within the dashed frame in the picture, share the common low side of the HV bias.

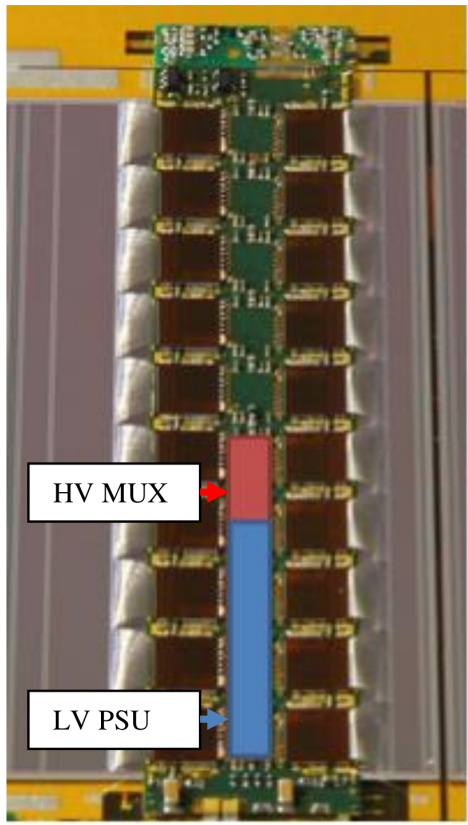

Figure 3. Proposed allocated area, of approximately $10 \times 10 \mathrm{~mm}^{2}$, for the HV MUX on stave hybrid.

strip detector modules are glued directly to each side of a composite support carbon fibre structure, which embeds the cooling pipe, figure 1 . The routing of signals and power between the modules and the End Of Stave (EoS) board, that acts as interface to the stave and is located at one end of the stave, is provided by a long kapton flex circuit, known as Bus Tape, that is glued to the carbon fibre skin of the stave. The schematic diagram of the HV MUX approach is shown in figure 2 with reference to a serial powering scheme, but it would equally be valid for a DC-to-DC powering scheme. Each HV switch is individually addressable through a DCS signal and permits enabling or disabling of the HV biasing to a sensor. When in the off mode, the HV switches should be able to withstand in excess of $500 \mathrm{~V}$, at the same time exhibiting much smaller leakage current than the sensors operating under normal condition (few 100's nA when non irradiated). Conversely, when in the on mode the HV switches should present a relatively low resistance, to allow the flow of up to around $8 \mathrm{~mA}$, owing to the increased leakage of the sensors after years of exposure to radiation. The predictions for the maximum fluence and dose in the strip barrel detectors are $5.3 \cdot 10^{14} 1 \mathrm{MeV}$ $\mathrm{n}_{\mathrm{eq}} / \mathrm{cm}^{2}, 216 \mathrm{kGy}$ for the short strips in layer 1 and $8.1 \cdot 10^{14} 1 \mathrm{MeV} \mathrm{n} \mathrm{n}_{\mathrm{eq}} / \mathrm{cm}^{2}, 288 \mathrm{kGy}$ for the strip end cap [6]. A specification of $2 \cdot 10^{15} 1 \mathrm{MeV} \mathrm{n}$ eq $/ \mathrm{cm}^{2}$ maximum fluence is therefore imposed to allow for uncertainties in fluence calculations, which sets the targeted radiation tolerance of the 
HV switches. Moreover, the HV switches should be able to operate in a strong magnetic field of around $2 \mathrm{~T}$, at low temperature $\left(\sim-30^{\circ} \mathrm{C}\right)$ and to be economically viable, as the estimated number of devices needed is in excess of 10,000.

The proposed allocated area for the implementation of the HV MUX is on the hybrid of the strip module, figure 3, where a small PCB carrier would include the LV powering, the HV MUX and the current measuring circuitry. This poses an additional requirement to the maximum size of HV switches of the order of a few 10 's $\mathrm{mm}^{2}$.

\subsection{Devices irradiation test description}

The challenging requirements for the HV switches outlined above make it difficult to identify readily available silicon devices suitable for this application. A number of different semiconductor devices, based upon silicon but also wider band gap material, like $\mathrm{SiC}$ and $\mathrm{GaN}$, have been thoroughly investigated to assess their suitability to this task.

In order to gain a better insight into the behaviour of different devices when exposed to these high levels of dose of radiation, a dedicated system capable of measuring in real time the electrical characteristics of the devices has been set up and used during irradiation tests performed at University of Birmingham of $26 \mathrm{MeV}$ proton irradiation facility, figure 4. It allows $\mathrm{HV}$ biasing and current measuring of the DUTs during the irradiation tests so as to monitor their changes at various level of fluence. For the test, the DUTs are wire bonded and glued on a PCB carrier, in turn plugged in, via a ZIF socket, to a PERSPEX frame, that allows XY adjustment of the PCB. This permits an easy replacement of different type of DUTs and fine adjustment of their positioning with respect to the $10 \times 10 \mathrm{~mm}^{2}$ size radiation beam, figure 5 .

An example of an irradiation sequence performed during the latest test of GaN devices EPC2012, rated for 200V, is shown in figure 6. After an initial transient of a few minutes (yellow) the DUTs are turned ON, by applying a Vgs $=3 \mathrm{~V}$. A constant current of up to $10 \mathrm{~mA}$, of a specific value to reflect the expected leakage currents of silicon sensors after years of operation at HL-LHC, is then injected into their drains and the resulting $\mathrm{V}_{\mathrm{ds}}$ is monitored. The beam is then turned on (red) while the $\mathrm{V}_{\mathrm{ds}}$ of the DUTs is continuously being monitored (blue). After 0.5 minute the beam is turned off, the DUTs are turned off and a high voltage bias is applied to their drain. A voltage sweep of up to $3 \mathrm{~V}$ in steps of $50 \mathrm{mV}$ is then applied to their gate and the resulting $\mathrm{I}_{d}$ and $\mathrm{I}_{g}$ are monitored. After the first $\mathrm{I}_{\mathrm{ds}}$ plot is completed, the next cycle of irradiation takes place and the entire sequence is repeated until the DUTs are irradiated to the maximum fluence of $0.75 \cdot 10^{15}$ $\mathrm{p}^{+} / \mathrm{cm}^{2}$. For silicon, this would correspond to a fluence of approximately $2.25 \cdot 10^{15} 1 \mathrm{MeV} \mathrm{n}$ eq $/ \mathrm{cm}^{2}$, more than the actual maximum expected fluence for the strips sensors in the upgraded tracker of ATLAS. The fractionated irradiation sequence allows measuring the change of various parameters of interest of the DUTs, including their leakage current when HV biased and their $r_{d s}$ when turned on, at different level of dose of radiation. Also the fractionated delivery safely prevents overheating of the DUTs as a result of the intense energy deposition during the irradiation phases. An example plot of real time measurements during the irradiation of the DUTs is shown in figure 7.

The DUTs are initially kept off, with the HV bias (150V, green curve) applied and the measured $I_{d s}$ is very low (black and red curves). When the applied $V_{g s}$, which sweeps between 0 and $3 \mathrm{~V}$ in steps of $50 \mathrm{mV}$, reaches the treshold value of the DUT the $\mathrm{I}_{\mathrm{ds}}$ starts to increase up to the maximum compliance value of $1 \mathrm{~mA}$ and, correspondingly, the $\mathrm{V}_{\mathrm{ds}}$ drops, eventually reaching a saturation 

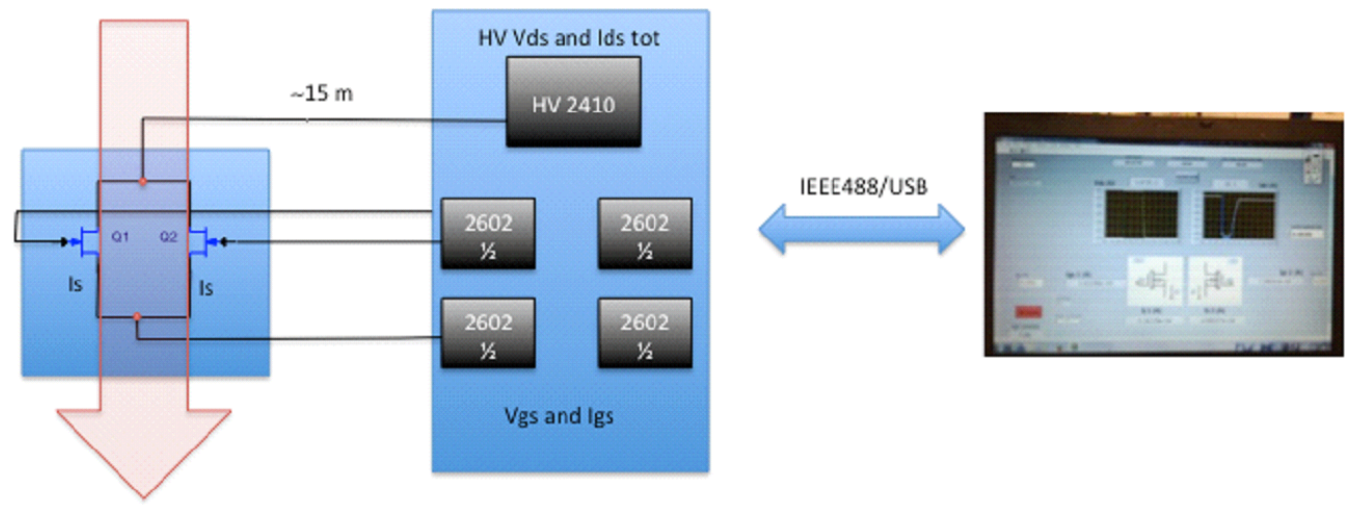

Figure 4. Diagram of the systems that allows real time measurement of the DUTs parameters during irradiation.
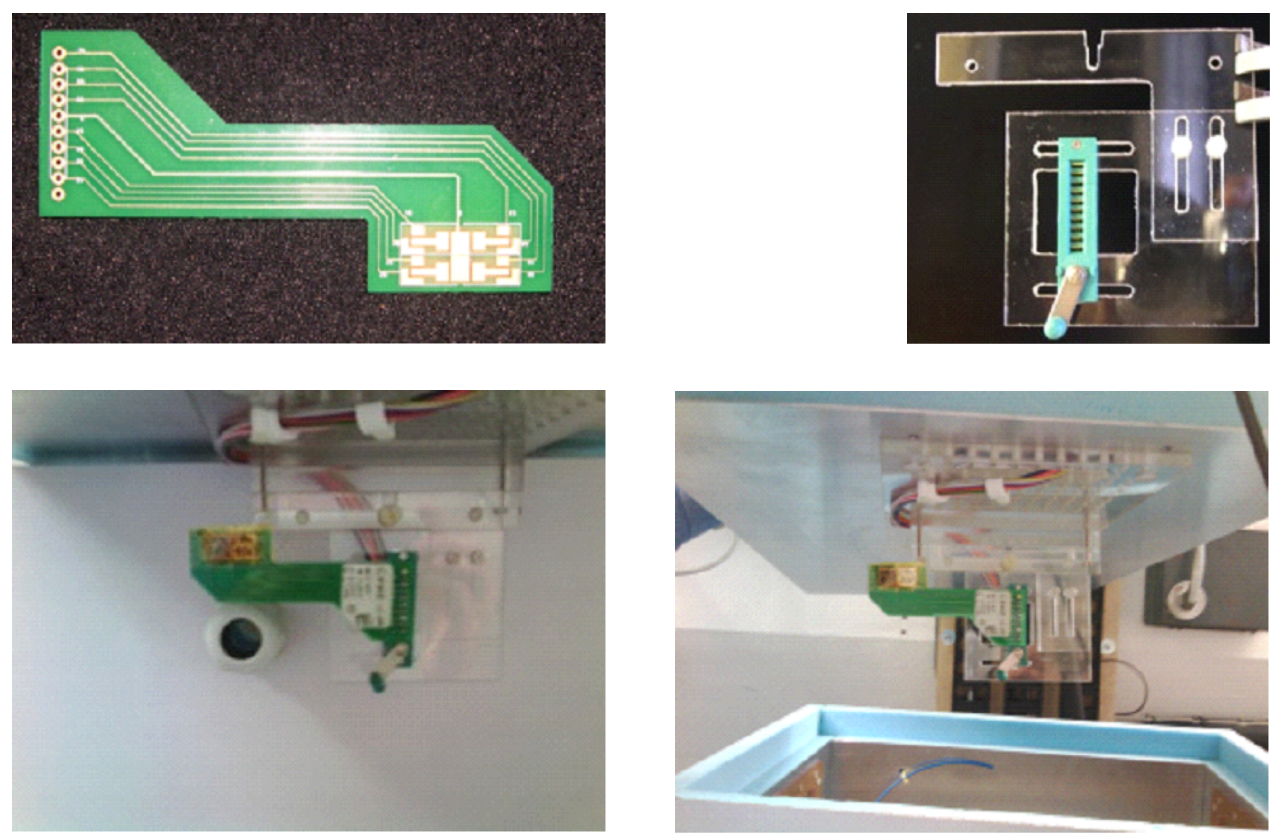

Figure 5. PCB carrier to hold the DUTs (top left), PERSPEX frame (top right) and positioning inside the cool box for radiation test at Birmingham (bottom left and right).

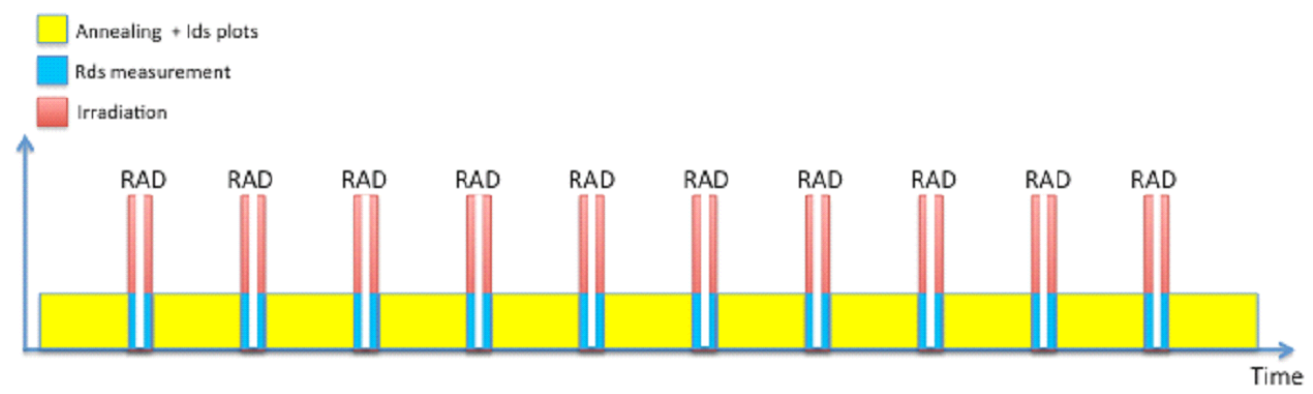

Figure 6. The irradiation sequence for DUTs radiation test. 


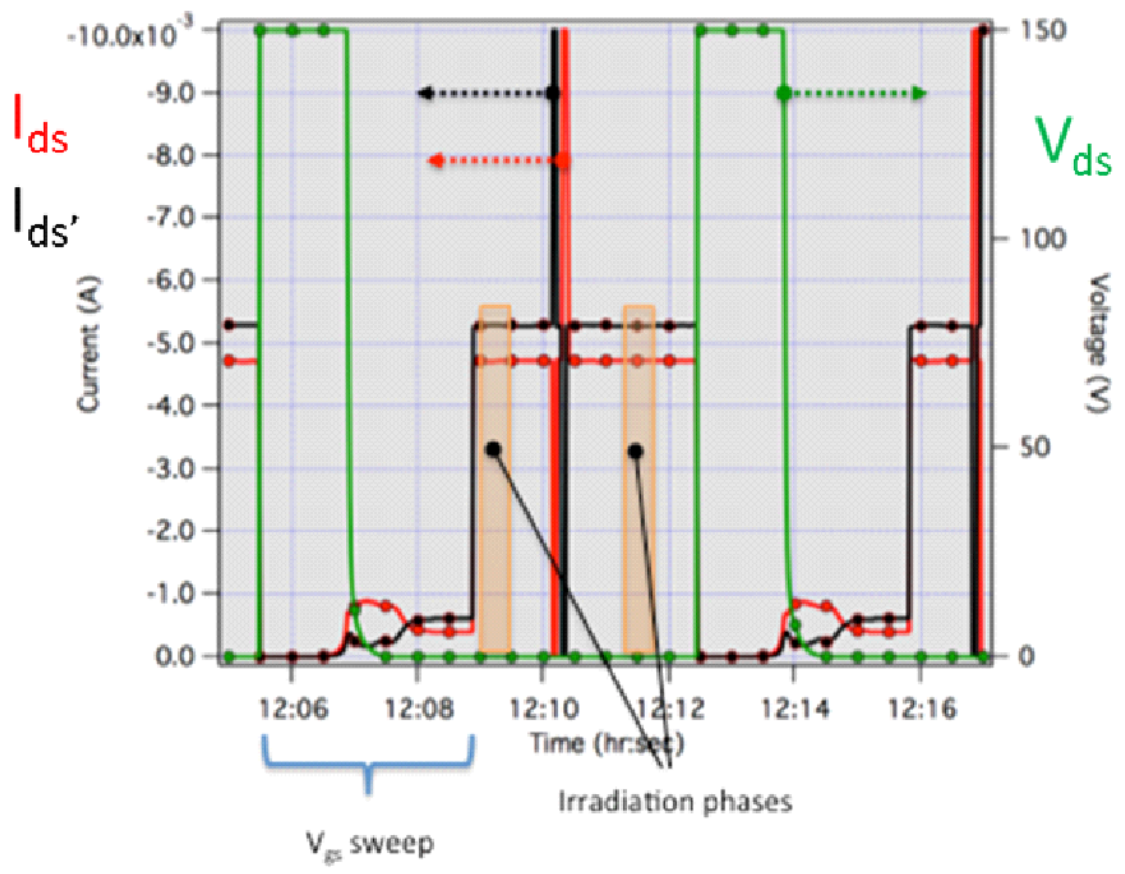

Figure 7. Example of $\mathrm{V}_{\mathrm{ds}}$ and $\mathrm{I}_{\mathrm{ds}}$ measurements during the real time irradiation of EPC2012.

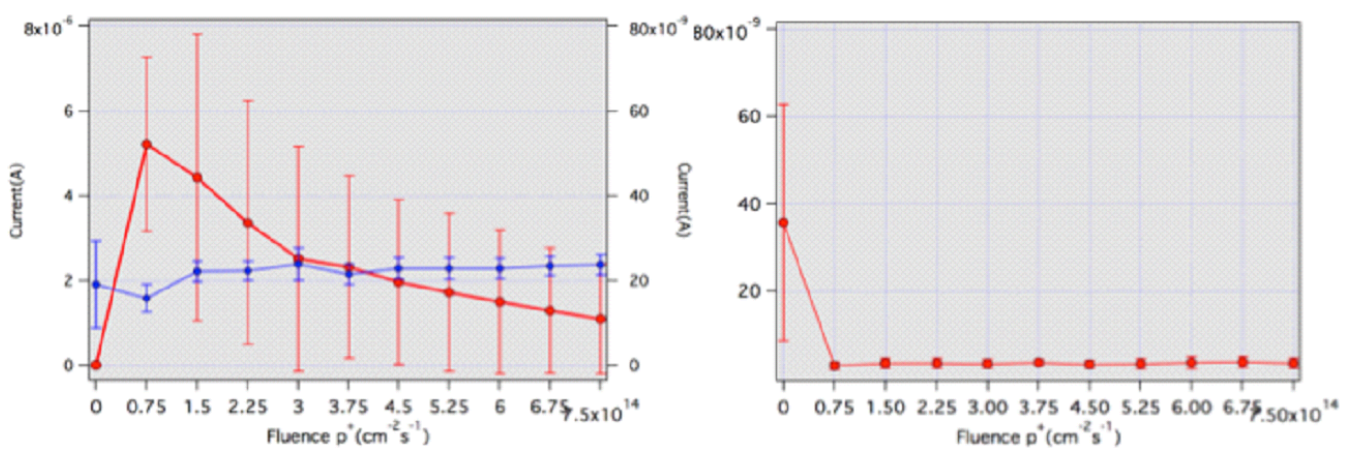

Figure 8. Plots of $I_{d s}$ and $I_{g s}$ change vs. fluence of EPC2012 in the off state with $V_{d s}=150 V$ and $V_{g s}=0 V$ (left), along with $\mathrm{I}_{\mathrm{gs}}$ plots vs. fluence in the on state with $\mathrm{V}_{\mathrm{gs}}=3 \mathrm{~V}$ (right).

value. Then the DUTs are kept on, with a $\mathrm{V}_{\mathrm{gs}}=3 \mathrm{~V}$, their $\mathrm{I}_{\mathrm{ds}}$ increases to the maximum value of the injected current, $10 \mathrm{~mA}$, and the $\mathrm{V}_{\mathrm{ds}}$ drops. Any change in $\mathrm{I}_{\mathrm{ds}}$ leakage or $\mathrm{V}_{\mathrm{ds}}$ increase during these phases is then monitored by the control software. Test results of the four irradiated EPC2012 devices so far show very little change in leakage current during $\mathrm{HV}$ bias and in resistivity during the on phases, suggesting that $\mathrm{GaN}$ devices could potentially be good candidates for this application, figure 8 .

\subsection{Stacked configuration for Higher Voltages}

Despite some of the investigated devices being rated for lower voltage than what would be needed for this application, it may be possible to use lower voltage devices in a stacked configuration, figure 9. 


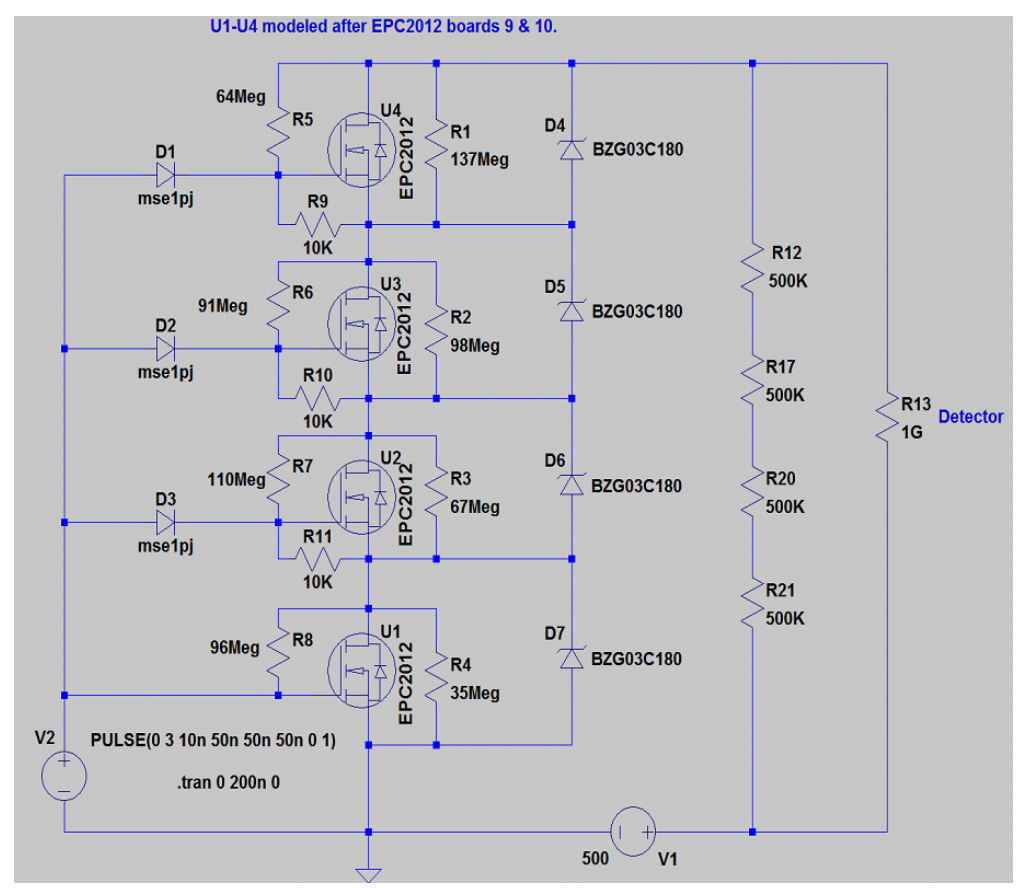

Figure 9. Stacked configuration of EPC 2012 devices, employing up to four devices, individually rated for up to $200 \mathrm{~V}$, to achieve $600 \mathrm{~V}$ switching capabilities.
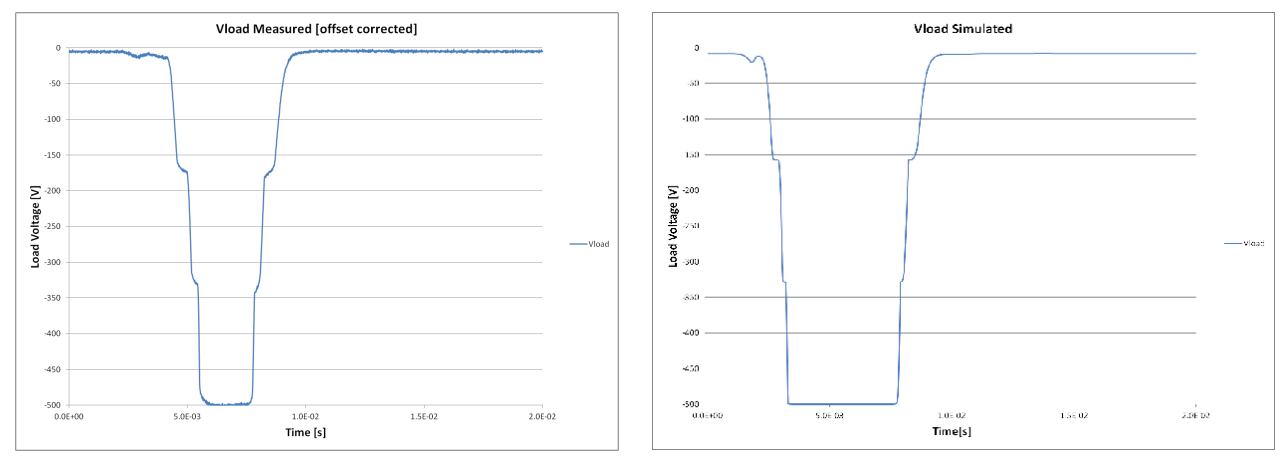

Figure 10. Simulated (left) and measured (right) results of the proposed stacked configuration of EPC 2012 devices, employing up to four devices, individually rated for up to $200 \mathrm{~V}$, to achieve $600 \mathrm{~V}$ switching capabilities.

Using a combination of resistors and diodes to guaranteee the proper biasing of the devices and that their maximum rated voltage is not exceeded, preliminary results on a test circuit validate this approach, figure 10. A proper circuit layout to satisfy the requirements of the allowed size of the HV MUX is currently being designed.

\section{Conclusions}

The HV bias distribution in the ATLAS Tracker upgrade will require sharing of the HV line among several sensors. In order to maintain an acceptable level of robustness an approach that uses HV switches to perform bias multiplexing is currently being investigated. 
A number of HV devices, based on silicon and wide band gap materials, are currently being investigated for this purpose. Electrical and radiation tests have been performed, suggesting that GaN based devices are potentially capable of fulfilling the requirements of this project. Additional tests will be performed soon to confirm the suitability of these devices to the intended application and to establish their sensitivity to single event effects.

A stacked circuitry that allows to use low voltage rated device to achieve higher voltage switching has been investigated and initial test results confirm the viability of such approach.

The next step in this project will be the confirmation of the radiation hardness of the GaN devices along with a dedicated layout to satisfy the area allocation requirements.

\section{Acknowledgments}

The authors would like to thank John Wilson, Paul Hodgson and Paul Dervan for their support during the irradiation tests at University of Birmingham.

\section{References}

[1] J. Bohm et al., Power supply and power distribution system for the ATLAS silicon strip detectors, in the proceedings of the $7^{\text {th }}$ Workshop on Electronics for LHC Experiments, September 10-14, Stockholm, Sweden (2001), CERN-2001-005.

[2] P. Phillips, ATLAS SCT power Supply System, in the proceedings of Topical Workshop on Electronics for Particle Physics 2007 (TWEPP2007), September 3-7, Prague, Czech Republic (2007).

[3] ATLAS collaboration ATLAS letter of intent Phase-II Upgrade for the ATLAS experiment, CERN-LHCC-2012-022 (2013).

[4] M. Elsing and P. Wells, Performance specifications of the Tracker Phase-II Upgrade, ATL-UPGRADE-PUB-2012-003 (2012).

[5] A. Clark et al., Final report: ATLAS Phase-II Tracker Upgrade layout task force, ATL-UPGRADE-PUB-2012-004 (2012).

[6] P.S. Miyagawa and I. Dawson, Radiation background studies for the Phase-II inner tracker upgrade, ATL-UPGRADE-INT-2012-012 (2012). 